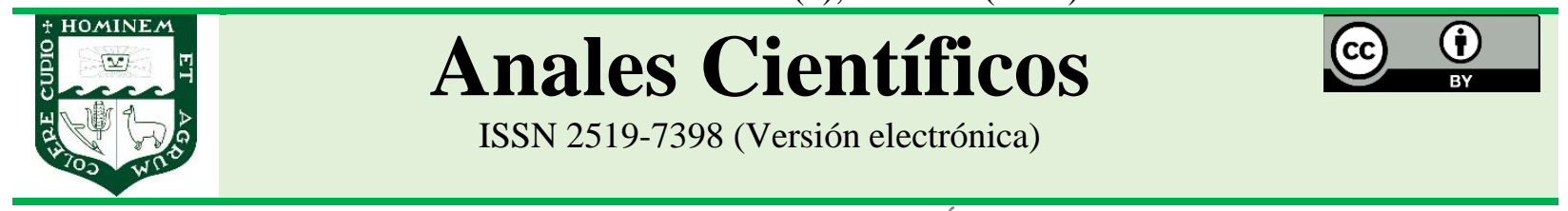

ARTÍCULO ORIGINAL - RESEARCH ARTICLE

http://dx.doi.org/10.21704/ac.v81i2.1644

\title{
EFECTO DE LA APLICACIÓN COMBINADA DE ABONO LÍQUIDO Y SÓLIDO EN LA PRODUCCIÓN DE PLANTINES DE CAFÉ (Coffea arabica) CULTIVADO BAJO CONDICIONES DE VIVERO EN CHIRINOS, CAJAMARCA, PERÚ
}

\section{Effect of new solid and liquid fertilizers on the production of coffee (Coffea arabica) seedlings under nursery cultivation in Chirinos, Cajamarca, Peru}

\author{
Juan Jaulis C. ${ }^{1 *} \mathbb{D}$; Ana Martínez V ${ }^{2}$; Juan Juscamaita M ${ }^{1}$; Enrique Adama Rojas ${ }^{1}$; Jorge Adama \\ Goméz $^{2}$
${ }^{1}$ Facultad de Agronomía, Universidad Nacional Agraria La Molina, 15024, Lima, Perú.
${ }^{2}$ Investigador independiente, Lima, Perú.
*E-mail: jjaulis@lamolina.edu.pe

\begin{abstract}
The obtaining of vigorous plants in nursery guarantees the successful development of crops in the field. Fertilization of plants in nursery is one of the fundamental pillars in the establishment of crops that can remain for more than 15 years in the field. In this regard, the objective of this study was to determine the effect of organic fertilizers Churufer and Churubiol made in Finca Churupampas Chirinos-Cajamarca-Peru for the production of coffee seedlings (Yellow Caturra variety) from nursery. The experiment was conducted as a Randomized Complete Block Design (RCBD) with sixteen treatments, three blocks and ten plants per experimental unit. The organic fertilizers used were $T_{1}$ ) Control without organic fertilizer, $T_{2}$ ) Churufer (biosol), $T_{3}$ ) Pachacushi (compost), $T_{4}$ ) Guano Island, $T_{5}$ ) Churubiol (biol), $\mathrm{T}_{6}$ ) Kapsitron (biol), $\mathrm{T}_{7}$ ) Huallquin-fer (biol), $\mathrm{T}_{8}$ ) Churufer+Churubiol, $\mathrm{T}_{9}$ ) Churufer+Kapsitron, $\mathrm{T}_{10}$ ) Churufer+Huallquin-fer, $\mathrm{T}_{11}$ ) Pachacushi+Churubiol, $\mathrm{T}_{12}$ ) Pachacushi+Kapsitron, $\mathrm{T}_{13}$ ) Pachacushi+Huallquinfer, $\mathrm{T}_{14}$ ) Guano Island+Churubiol, $\mathrm{T}_{15}$ ) Guano Island+Kapsitron, $\mathrm{T}_{16}$ ) Guano Island +Huallquin-fer. After 120 days, it was verified that coffee seedlings treated with the combination of organic fertilizers Churufer+Churubiol presented the best results in Plant Height $(15,64 \mathrm{~cm})$, Number of Leaves $(8,83)$, Stem Diameter $(2,62 \mathrm{~mm})$, Fresh Weight of Leaves $(3,09 \mathrm{~g})$, Fresh Weight of Stem $(0,0054 \mathrm{~g})$, Fresh Weight of Root $(0,0100 \mathrm{~g})$ and Total Fresh Weight $(3,0988 \mathrm{~g})$. In this way, it is concluded that the organic fertilizers elaborated in Finca Churupampas significantly improved the size and weight of coffee seedlings.
\end{abstract}

Keywords: organic fertilization; homolytic fermentation; slow release fertilizer pellets; slow release fertilizers

\section{RESUMEN}

La obtención de plantas vigorosas en vivero garantiza el desarrollo exitoso de cultivos en el campo definitivo, para ello la fertilización de plantas en vivero es uno de los pilares fundamentales en el establecimiento de cultivos que pueden permanecer por más de 15 años en campo. En ese sentido, el objetivo de este estudio fue evaluar el efecto combinada de los abonos orgánicos Churufer y Churubiol, elaborados en la Finca Churupampas Chirinos- 
Cajamarca-Perú, en la producción de plantas de café (variedad Caturra amarillo) a nivel de vivero. El experimento fue conducido mediante un Diseño de Bloques Completamente al Azar (DBCA) con dieciséis tratamientos, tres bloques y diez plantas por unidad experimental. Los abonos utilizados fueron: $T_{1}$ ) Testigo sin fertilizante orgánico, $\mathrm{T}_{2}$ ) Churufer (biosol), $\mathrm{T}_{3}$ ) Pachacushi (compost), $\mathrm{T}_{4}$ ) Guano de Isla, $\mathrm{T}_{5}$ ) Churubiol (biol), $\mathrm{T}_{6}$ ) Kapsitron (biol), $\mathrm{T}_{7}$ ) Huallquin-fer (biol), $\mathrm{T}_{8}$ ) Churufer+Churubiol, $\mathrm{T}_{9}$ ) Churufer+Kapsitron, $\mathrm{T}_{10}$ ) Churufer+Huallquin-fer, $\mathrm{T}_{11}$ ) Pachacushi+Churubiol, $\mathrm{T}_{12}$ ) Pachacushi+Kapsitron, $\mathrm{T}_{13}$ ) Pachacushi+Huallquin-fer, $\mathrm{T}_{14}$ ) Guano de Isla+Churubiol, $\mathrm{T}_{15}$ ) Guano de Isla+Kapsitron, $\mathrm{T}_{16}$ ) Guano de Isla+Huallquin-fer. Después de 120 días, fue verificado que plantas de Café en vivero tratadas con la combinación de fertilizantes orgánicos Churufer+Churubiol presentaron los mejores resultados en Altura de planta $(15,64 \mathrm{~cm}), \mathrm{N}^{\circ}$ Hojas $(8,83)$, Diámetro de Tallo $(2,62 \mathrm{~mm})$, Peso Fresco de Hojas (3,09 g), Peso Fresco de Tallo (0,0054 g), Peso Fresco de Raíz (0,0100 g) y Peso Fresco total (3,0988 g). De esta manera, se concluye que los fertilizantes orgánicos elaborados en la Finca Churupampas mejoraron significativamente el tamaño y peso de plantas de café en vivero.

Palabras clave: Fertilización orgánica; fermentación homoláctica; pellets de lenta liberación; abonos de lenta liberación

Forma de citar el artículo (Formato APA):
Jaulis, J., Martínez, A., Juscamaita, J., Adama, E. \& Adama, J. (2020). Efecto de la aplicación combinada de abono líquido y
sólido en la producción de plantines de café (Coffea arabica) cultivado bajo condiciones de vivero en Chirinos, Cajamarca,
Perú. Anales Científicos. 81(2), 336-346. http://dx.doi.org/10.21704/ac.v81i2.1644
Autor de correspondencia (*): Juan Carlos Jaulis C. Email: jjaulis@ lamolina.edu.pe
@ Los autores. Publicado por la Universidad Nacional Agraria La Molina.
This is an open access article under the CC BY

\section{INTRODUCCIÓN}

La planta de café (Coffea arabica L.) pertenece a la familia Rubiaceae la cual es la cuarta familia más grande dentro de las plantas angiospermas, con un número total estimado de aproximadamente 13,000 especies dentro de 650 géneros (Koehbach y Gruber, 2015). Es una planta oriunda de Etiopía y su introducción al Perú se estima en el año 1760 (Barrenechea, 1986).

El café es un cultivo de alta importancia económica para el Perú por ser uno de los principales productos agrícolas de exportación que contribuye a la generación de ingresos y empleo para el país (Castrillón et al., 2017). De las variedades sembradas en el Perú el 70\% corresponde a la variedad Typica, seguido de Caturra con un $20 \%$ y otras con un $10 \%$ (USDA, 2018).

Actualmente, en el país existen 16 regiones productoras de café, entre las principales regiones se encuentran San Martín, Junín, Cajamarca, Amazonas y Cusco. Para el año 2017, se estimó un total de 384 mil hectáreas cosechadas de café y se registró 236 mil familias productoras de este grano (MINAGRI, 2018; MINCETUR, 2018). Perú con 90 mil hectáreas con certificación orgánica, es el líder en exportaciones de café orgánico. Asimismo, una importante porción de café exportado de Perú es orgánico debido en gran parte a la incapacidad de los pequeños productores de pagar por costosos fertilizantes químicos y pesticidas (USDA, 2018).

Las necesidades del sector cafetalero de incrementar la eficiencia productiva acompañado con la reducción de costos de producción para incrementar la competitividad hacen que sea necesario la búsqueda de nuevas alternativas de producción y nuevos suministros para el desarrollo de una agricultura sostenible (Evangelista et al., 2013; Maradiaga et al., 2017). El alto costo económico y ambiental de la agricultura convencional ha hecho que muchos agricultores repiensen su forma de producción y opten por el uso de los desperdicios orgánicos como alternativa viable y sostenible para la fertilización (Rezende et al., 2010; Pindi, 2012).

La fertilización orgánica consiste en la aplicación de compuestos orgánicos elaborados a partir de residuos de origen vegetal y animal al suelo y/o a las plantas (Medeiros et al., 2008). Entre algunas fuentes naturales que pueden usarse para la producción de café orgánico se encuentran el Guano de Islas, el Compost, los bioles y los biosoles. Estos productos son conocidos 
comercialmente y su uso está permitido por la normatividad nacional en el D.S. $\mathrm{N}^{\circ}$ 044-2006-AG (SENASA, 2006) e internacional para este rubro (USDA, 2013).

El Guano de Islas es el producto proveniente de la alimentación de las aves marinas como Guanay (Phalacrocorax boungainvilli), Piquero (Sula variegata) y Pelicano (Pelecanus thagus) con especies hidrobiológicas como la anchoveta, sardina y pejerrey, que luego de ser procesadas en sus aparatos digestivos, deyectan en las islas y puntas del litoral peruano, formando grandes reservas que en el transcurso de 5 a 7 años se convierten en unos de los mejores abonos naturales orgánicos del mundo debido a su alto contenido de nitrógeno y fósforo (Ceroni, 2012; Agro Rural, 2018).

El compostaje y el uso de biodigestores anaerobios son tecnologías agropecuarias comúnmente utilizadas por los agricultores para tratar los residuos orgánicos de origen vegetal, animal, agroindustrial, forestal, doméstico u otros, siendo alternativas económicas viables y ambientales al manejo de estos residuos (Carhuancho, 2012). El compostaje utiliza los desechos orgánicos y los convierte en compost por medio de microorganismos que trabajan en condiciones aeróbicas. Las propiedades físicas y químicas del compost pueden ser afectados por los tipos y números de microorganismos presentes en el proceso de compostaje (Cai et al., 2018). En cambio, los principales productos del proceso de digestión anaerobia de los biodigestores son el biogás, un producto energético y el bioabono, un efluente estabilizado. El bioabono obtenido luego de 3 a 4 meses puede presentarse de dos formas: líquida (biol) y sólida (biosol) que se caracterizan por ser ricos en elementos minerales (Varnero, 2011; Carhuancho, 2012). Sin embargo, la producción de biofertilizantes: biol y biosol también pueden ser obtenidos por medio de una fermentación homoláctica en condiciones anaeróbicas, que implica el uso de residuos orgánicos de diversas industrias, melaza y del consorcio microbiano B-lac (consorcio de bacterias probióticas del género Lactobacillus). Esta fermentación realizada con el consorcio B-lac se caracteriza por ser una técnica más rápida en cuanto al tiempo de obtención del bioabono, con alto contenido de nutrientes, $\mathrm{pH}$ bajo y ausencia de coliformes fecales y otros patógenos adversos (Román,
2012; Buchelli, 2014; Meza, 2014; Medina et al., 2015; Peralta et al., 2016; Quiñones et al., 2016; Herrera, 2017; Aguirre, 2017; Cupe y Juscamaita, 2018).

Diversos autores (Marcuzzo et al., 2005; Borjas, 2008; Maradiaga et al., 2017; Pinedo et al., 2018) concuerdan en que las aplicaciones de estos fertilizantes orgánicos intervienen en la producción de plantas de calidad ya que pueden afectar el desarrollo y crecimiento de plantas en vivero y en su futura implantación al campo de cultivo definitivo.

En la necesidad de generar tecnologías de fertilización orgánica para pequeños productores cafetaleros y asegurar su éxito en la agricultura familiar es que se desarrolló un abono líquido procedente de la fermentación homoláctica de residuos, abonos orgánicos y el consorcio microbiano B-lac (Churubiol) y un abono sólido de lenta liberación en forma de pellets constituido por residuos, abonos orgánicos y agentes aglomerantes (Churufer), ambos con alto contenido de Nitrógeno $(\mathrm{N})$, Fósforo $\left(\mathrm{P}_{2} \mathrm{O}_{5}\right)$ y Potasio $\left(\mathrm{K}_{2} \mathrm{O}\right)$. Churufer y Churubiol fueron comparados con fertilizantes orgánicos comúnmente empleados en la agricultura orgánica en el distrito de ChirinosCajamarca.

Es así que este trabajo tuvo como objetivo de estudio determinar el efecto de los fertilizantes orgánicos Churufer y Churubiol en la producción plantas de café a nivel de vivero.

\section{MATERIALES Y MÉTODOS}

La investigación se desarrolló durante el periodo setiembre a diciembre del 2018 bajo condiciones de vivero en la Finca Churupampa, ubicado en el Distrito Chirinos, Provincia de San Ignacio, Región Cajamarca, a una Altitud de $1372 \mathrm{msnm}$, con coordenadas geográficas de S $5^{\circ} 18,27.4^{\prime \prime}$ y W 78 52,56.4”. Durante la investigación las temperaturas oscilaron entre $19^{\circ} \mathrm{C}$ y $25^{\circ} \mathrm{C}$.

\section{Instalación}

Para la instalación del trabajo de investigación se seleccionó $1 \mathrm{~kg}$ de semilla de café (Coffea arabica L.) de la variedad Caturra amarillo procedentes de parcelas del mismo lugar. La germinación de las semillas de café se dio a los 60 días y el repique se realizó en palito de fósforo en bolsas de polietileno de 4"x8" con tierra- $y$ 
abono tipo bocashi en una relación 3:1 como mezcla de sustrato. Las plántulas seleccionadas fueron de tamaños similares, sanas y bien formadas.

Luego del transplante, tres tipos de abonos sólidos (Churufer, Guano de Islas, Pachacushi) y tres tipos de bioles (Churubiol, Huallquin-fer y Kapsitron) fueron utilizados solos o en combinación para su aplicación en los plantines. Es así que el experimento fue conducido mediante un Diseño de Bloques Completamente al Azar (DBCA) con dieciséis tratamientos, tres bloques y diez plantas por unidad experimental. Los tratamientos empleados fueron los siguientes: $\mathrm{T}_{1}$ ) Testigo sin biofertilizante, $\mathrm{T}_{2}$ ) Churufer, $\mathrm{T}_{3}$ ) Pachacushi, $\mathrm{T}_{4}$ ) Guano de Isla, $\mathrm{T}_{5}$ ) Churubiol, $\mathrm{T}_{6}$ ) Kapsitron, $\mathrm{T}_{7}$ )Huallquin-fer, $\mathrm{T}_{8}$ ) Churufer+Churubiol, $\mathrm{T}_{9}$ ) Churufer + Kapsitron, $\mathrm{T}_{10}$ ) Churufer+Huallquin-fer, $\mathrm{T}_{11}$ ) Pachacushi + Churubiol, $\mathrm{T}_{12}$ ) Pachacushi + Kapsitron, $\mathrm{T}_{13}$ ) Pachacushi + Huallquin-fer, $\left.\mathrm{T}_{14}\right)$ Guano de Isla + Churubiol, $\mathrm{T}_{15}$ )Guano de Isla + Kapsitron, $\mathrm{T}_{16}$ ) Guano de Isla+Huallquin-fer.

\section{Descripción de los abonos sólidos}

Churufer es un abono sólido de lenta liberación en forma de pellets constituido por insumos de procedencia orgánica. Mejora las propiedades físicas, químicas y biológicas del suelo al mismo tiempo aporta nutrientes como $\mathrm{N}(12,45), \mathrm{P}(8,81), \mathrm{K}(5,98)$ y $\mathrm{Ca}$ $(8,94)$.

Guano de Islas proviene de los restos y excrementos de las aves guaneras (Guanay, Piquero y Pelicano) que habitan las islas y puntas del litoral peruano. Dentro de las propiedades del guano de islas se tiene que es un fertilizante natural y completo, producto ecológico, biodegradable, mejora las propiedades físicas, químicas $\mathrm{y}$ biológicas del suelo, es soluble en agua y tiene propiedades de sinergismo.

Pachacushi es un fertilizante sólido orgánico utilizado en suelos pobres o deficientes en Materia Orgánica, Potasio, Magnesio y Boro. Aporta una importante fracción de materia orgánica a los cultivos con un $\mathrm{pH}$ neutro.

\section{Descripción de los abonos líquidos}

Churubiol es un biol constituido por residuos de la producción del café, más un consorcio microbiano Blac (consorcio de bacterias probióticas del género Lactobacillus) y abonos orgánicos, los cuales pasan por un proceso de fermentación homoláctica en condiciones anaeróbicas.

Hualquin-Fer es un biofertilizante líquido orgánico que contiene fitobióticos y nutrientes para las plantas. Es obtenido por un proceso biotecnológico de fermentación homoláctica de excretas de ganado vacuno y suero lácteo dulce. Su aplicación al suelo y a la parte foliar, permite la disposición de nutrientes de muy fácil asimilación. Asimismo, mejora el $\mathrm{pH}$ del suelo, controla la presencia de mircroorganismos (hongos y bacterias), nematodos y otros fitopatógenos.

Kapsitron es un fertilizante biológico a base de capsaina (activadores y precursores de fitoalexinas) con propiedades repelentes contra insectos picadores chupadores.

La composición química de los insumos individuales es presentada en la Tabla 1.

Los abonos sólidos fueron aplicados a razón de 20 gramos por planta a los 25 días después de la siembra. Los bioles fueron aplicados con mochila de aspersión a razón de $10 \mathrm{ml}$ por 1 litro de agua al follaje a los 30 días después de la siembra.

\section{Labores de mantenimiento}

El riego fue diario y el sustrato fue mantenido a capacidad de campo. El agua utilizada provino de la vertiente Churupampa.

\section{Evaluación}

Las plantas de café en vivero fueron evaluadas a los tres meses después de la siembra. Se evaluaron los siguientes parámetros: Altura Total de Planta $(\mathrm{cm})$, Número de Hojas, Diámetro de Tallo (mm), Peso Fresco de Hojas (g), Peso Fresco de Raíz (g), Peso Fresco Total (g). Las variables evaluadas se analizaron con el paquete estadístico Statistical Analysis System (SAS) versión 9.4. El procesamiento de datos se realizó con el Análisis de Varianza (ANVA). Para determinar la comparación de medias se utilizó la prueba de rango múltiple de DUNCAN considerando un nivel de significancia de 0,05 . 


\section{RESULTADOS Y DISCUSIÓN}

Los resultados del análisis estadístico son presentados en la Tabla 2. Puede observarse que en todas las variables en estudio: Altura de planta $(15,64 \mathrm{~cm}), \mathrm{N}^{\circ}$ Hojas (8,83), Diámetro de Tallo $(2,62 \mathrm{~mm})$, Peso Fresco de Hojas (3,09 g), Peso Fresco de Tallo (0,0054 g), Peso Fresco de Raíz (0,0100 g) y Peso Fresco total $(3,0988 \mathrm{~g})$, el tratamiento Churufer+Churubiol $\left(\mathrm{T}_{8}\right)$ fue estadísticamente el mejor en comparación a los demás biofertilizantes y al testigo que no tuvo aplicación alguna de bioabonos.

La combinación de Churufer y Churubiol $\left(\mathrm{T}_{8}\right)$ obtuvo los mejores resultados de producción de plantines de café en vivero debido a que el abono sólido (Churufer) aporto un alto contenido de nutrientes $(12,45 \% \mathrm{~N}$, $\left.8,81 \% \quad \mathrm{P}_{2} \mathrm{O}_{5}, 5,98 \mathrm{~K}_{2} \mathrm{O}\right)$ al medio de crecimiento, en forma de pellets de lenta liberación. Los pellets en su composición contenían agentes aglomerantes como goma de tara, carragenina y almidón de yuca, los cuales permitieron que los nutrientes se liberaran en forma paulatina durante el ciclo de cultivo evaluado. La goma de tara es un compuesto que actúa espesando soluciones acuosas y controlando la movilidad de materiales dispersados o disueltos. Posee la ventaja de ser muy estable y altamente resistente a la descomposición (Apaza, 2018; MINAGRI, 2019). En el caso de la carragenina, una goma proveniente de extractos de algas (Mancilla, 2012), permite dar soluciones muy viscosas cuando es mezclada con la goma de tara (Belitz y Grosch, 1997). Finalmente, el almidón de yuca tiene diferentes aplicaciones industriales entre ellas como ligante de agua, coadyuvante de emulsificantes, fuente de carbohidratos, espesante y agente texturante (Montoya, 2007). Asimismo, el abono líquido (Churubiol) aportó a las plantas de café un alto contenido de nutrientes $\left(2,00 \% \mathrm{~N}, 1,10 \% \mathrm{P}_{2} \mathrm{O}_{5}, 1,50\right.$ $\mathrm{K}_{2} \mathrm{O}$ ) lo cual permitió tener un efecto más eficiente en la absorción de los nutrientes. Estos resultados concuerdan con los obtenidos por Arcáyo (2018) quien probó fertilizantes de lenta liberación en forma de pellets en el cultivo de maíz, encontrando que la liberación de nutrientes en el suelo se dio en forma paulatina, minimizando pérdidas por volatilización o infiltración. De la misma forma, Medeiros y Lopes (2006) y Bonten et al. (2014) mencionan que el aporte de nutrientes esenciales de un biofertilizante líquido permite tener un efecto más eficiente en la absorción de los nutrientes. El valor del biol como fertilizante es bastante alto, debido a que contiene nutrientes en una forma fácilmente disponible lo que significa que puede tener un mayor efecto en la fertilización en un corto plazo.

La disponibilidad de nutrientes en vivero es uno de los factores que determinan el éxito de la producción de plantones de café de alta calidad (Muliasari et al., 2015). Los principales nutrientes de las plantas que determinan el rendimiento y la calidad del café son $\mathrm{N}, \mathrm{P}_{2} \mathrm{O}_{5}$ y $\mathrm{K}_{2} \mathrm{O}$.

Tabla 1. Composición química de macronutrientes y micronutrientes en los diferentes biofertilizantes.

\begin{tabular}{llllllllll}
\hline $\begin{array}{c}\text { Tipo de } \\
\text { biofertilizante }\end{array}$ & \multicolumn{1}{c}{$\begin{array}{c}\mathrm{N} \\
(\%)\end{array}$} & $\mathrm{P}_{2} \mathrm{O}_{5}(\%)$ & $\mathrm{K}_{2} \mathrm{O}(\%)$ & $\begin{array}{c}\mathrm{CaO} \\
(\%)\end{array}$ & $\begin{array}{c}\mathrm{Fe} \\
\mathrm{ppm}\end{array}$ & $\begin{array}{c}\mathrm{Cu} \\
\mathrm{ppm}\end{array}$ & $\begin{array}{c}\mathrm{Mn} \\
\mathrm{ppm}\end{array}$ & $\begin{array}{c}\mathrm{B} \\
\mathrm{ppm}\end{array}$ & $\begin{array}{c}\mathrm{Zn} \\
\mathrm{ppm}\end{array}$ \\
\hline Churufer & 12,45 & 8,81 & 5,98 & 8,94 & 1918 & 16 & 109 & 43 & 9,20 \\
Guano de islas & $10,00-14,00$ & $10,00-12,00$ & $2,00-3,00$ & 8,00 & 320 & 240 & 200 & 160 & 20 \\
Pachacushi & 10,00 & 8,00 & 10,00 & 9,00 & - & - & - & 56700 & 500000 \\
Churubiol & 2,00 & 1,10 & 1,50 & - & - & - & - & - & - \\
Huallquin-fer & 0,24 & 0,35 & 0,19 & 0,29 & 144,1 & 2,24 & 27,23 & 3,04 & 6,63 \\
Kapsitron & 0,27 & 0,59 & 0,96 & 0,84 & 19,24 & 1,48 & 2,60 & 3,87 & 1,94 \\
\hline
\end{tabular}

Es así que el $\mathrm{T}_{8}$, proporcionó a las plantas de café en vivero nutrientes para su absorción vía el sistema radicular (Churufer) y vía foliar (Churubiol), asegurando de manera más eficiente la absorción de los nutrientes y logrando un mejor desarrollo vegetativo de las plantas
Estos resultados concuerdan con Malavolta (2000) quien afirma que, en vivero, la respuesta predominante es al nitrógeno y el fósforo, mientras que Carvajal (1984) hace referencia de que en campo la respuesta predominante de la planta es al 
nitrógeno y potasio. Shah et al. (2016) menciona que el nitrógeno en forma de $\mathrm{NO}_{3}-\mathrm{y} \mathrm{NH}_{4}+$ es el elemento más imperativo para el correcto crecimiento y desarrollo de las plantas, que aumenta y mejora significativamente el rendimiento y calidad al desempeñar un papel vital en las funciones bioquímicas y fisiológicas de las mismas. Asimismo, Arizaleta et al. (2002) afirma que en vivero las dosis más frecuentes de fertilizantes varían de 0,48 a 0,60 g de $\mathrm{N}$ y $\mathrm{K}_{2} \mathrm{O}$, y de 0,60 a 0,96 g de $\mathrm{P}_{2} \mathrm{O}_{5}$ por bolsa. Cai et al. (2007) afirma que cuanto mayor es la dosis de fertilizante que se administra a la planta de café, existe un mejor crecimiento vegetativo.

En la Tabla 1 puede observarse que el abono Guano de Isla presentó un alto contenido de nutrientes como el $\mathrm{N}(10 \%-14 \%)$ y $\mathrm{P}_{2} \mathrm{O}_{5}(10 \%-12 \%)$; sin embargo, el tratamiento $\mathrm{T}_{4}$ (Guano de Isla) solo fue estadísticamente igual a $T_{8}$ en cuanto a las variables $\mathrm{N}^{\circ}$ Hojas $(8,50)$, DTallo $(2,58 \mathrm{~mm})$ y PFRaíz $(0,0083 \mathrm{~g})$; esto puede deberse a que el abono guano de islas es susceptible a la perdida de nutrientes como el nitrógeno por medio de volatilización y lixiviación. En la combinación de abono guano de islas con la adición de algún biofertilizante líquido, se encontró que $\mathrm{T}_{14}$ (Guano de Isla+Churubiol) fue estadísticamente igual a $\mathrm{T}_{8}$ en las variables DTallo $(2,48 \mathrm{~mm})$ y PFTallo $(0,0063 \mathrm{~g}), \mathrm{T}_{15}$ (Guano de Isla+Kapsitron) fue estadísticamente igual a $\mathrm{T}_{8}$ en la variable PFRaíz $\left(0,0083\right.$ g) y $\mathrm{T}_{16}$ (Guano de Isla+Huallquin-fer) fue estadísticamente igual a $\mathrm{T}_{8}$ en la variable D Tallo (2,47 mm). Rojas (2017) encontró que la aplicación de guano de isla fue el tratamiento que mejores resultados obtuvo para plantas de café var. Caturra en vivero. Sin embargo, Pinedo et al. (2018) no mostró resultados superiores con Guano de Islas a pesar de su alta concentración de macronutrientes.

El proceso de fermentación homoláctica es un método de conservación de nutrientes en condiciones anaeróbicas, con el fin de promover la fermentación de azúcares a ácido láctico, lo cual causa un incremento de la acidez (Román, 2012; Buchelli, 2014; Meza, 2014; Medina et al., 2015; Peralta et al., 2016; Quiñones et al., 2016; Herrera, 2017; Aguirre, 2017; Cupe y Juscamaita, 2018). Los resultados obtenidos en la aplicación del abono sólido Churufer $\left(\mathrm{T}_{2}\right)$ a las plantas de café en vivero fueron estadísticamente similares a $\mathrm{T}_{8}$ en cuanto a las variables Altura de planta $(16,61 \mathrm{~cm}), \mathrm{N}^{\circ}$ Hojas $(8,70)$, D Tallo (2,73 mm), PF Hojas $(2,47 \mathrm{~g})$ y PF Raíz $(0,0087 \mathrm{~g})$ y estadísticamente diferente a $\mathrm{T}_{8}$ en las variables PF Tallo $(0,0047 \mathrm{~g})$ y PF Total $(2,4800$ g). Cupe y Juscamaita (2018) encontraron que el abono sólido (biosol) producido por fermentación homoláctica del lodo residual de la industria cervecera tuvo un contenido de nitrógeno elevado ya que no se volatilizó en forma de amoniaco porque el pH de dicho biosol fue ácido. Churufer mostró una buena calidad agronómica por su alto contenido de macro y micro nutrientes; asimismo, Buchelli (2014) afirma que el contenido de macronutrientes y micronutrientes del biosol producido en una fermentación homoláctica en comparación con otros biosoles resulta un producto con alto contenido nutricional. Ascue y Guerrero (1986) mencionan que la fermentación en depósitos cerrados evita pérdidas importantes de fósforo, potasio o calcio contenidos en la materia cruda.

En cuanto al compost Pachacushi $\left(\mathrm{T}_{3}\right)$, se encontró que su contenido de Nitrógeno $(10,00 \%)$ y Fósforo $(8,00 \%)$ fueron los más bajos de todos los abonos sólidos empleados. Los resultados evidenciaron que $\mathrm{T}_{3}$ fue estadísticamente igual a $\mathrm{T}_{8}$ en las variables $\mathrm{D}$ Tallo $(2,47 \mathrm{~mm})$ y PF Raíz $(0,0083 \mathrm{~g})$ y estadísticamente inferior a $\mathrm{T}_{8} \mathrm{e}$ igual a $\mathrm{T}_{1}$ en cuanto a las variables Altura de planta $(14,84 \mathrm{~cm}), \mathrm{N}^{\circ}$ Hojas $(8,11)$, PF Hojas $(2,08 \mathrm{~g})$, PF Tallo $(0,0047 \mathrm{~g})$ y PF Total $(2,0963 \mathrm{~g})$. Al no ser Pachacushi un fertilizante de lenta liberación y al tener un bajo contenido de Nitrógeno como abono sólido es que no demostró resultados superiores a $T_{8}$.

En tanto la aplicación del abono líquido Churubiol $\left(\mathrm{T}_{5}\right)$ fue estadísticamente igual a $\mathrm{T}_{8}$ en cuanto a las variables Altura de planta $(17,32 \mathrm{~cm}), \mathrm{N}^{\circ}$ Hojas $(8,9)$, D Tallo $(2,79 \mathrm{~mm})$ y PF Raíz $(0,0093 \mathrm{~g})$ y estadísticamente diferente a $\mathrm{T}_{8}$ en cuanto a PF Hojas $(2,37 \mathrm{~g})$, PF Tallo $(0,0047 \mathrm{~g})$ y PF Total $(2,3807 \mathrm{~g})$. Peralta et al. (2016) indicaron que la diferencia en el contenido de nitrógeno total entre bioles producidos de manera convencional y bioles basados en una fermentación homolactica, se debe al proceso de degradación de materia orgánica. Es así que Fastbiol, un biol producido por fermentación 
homolactica, presentó un mayor contenido de nitrógeno en forma nítrica $\left(\mathrm{N}-\mathrm{NO}_{3}{ }^{-}\right)$y amoniacal $(\mathrm{N}-$ $\mathrm{NH}_{4}^{+}$) en comparación con bioles producidos en forma artesanal.

Medina et al. (2015) encontraron que el biol producido por la digestión anaerobia en biodigestores (Biol I-G) y la fermentación homoláctica sobre este biol obtenido (Biol II-G) evidenciaron que el Biol II-G alcanzó valores de $\mathrm{pH}$ alrededor de 3.5, un nivel mucho más ácido con respecto al que presentó el Biol I-G (entre 6.8-7.8). Las concentraciones de nutrientes se incrementaron considerablemente al finalizar el proceso de producción del Biol II-G y muchos de los elementos hasta quintuplicaron sus valores. Los efluentes líquidos obtenido por Meza (2014) y Quiñones et al. (2016) denominados Papa-Biol y Alpa-biol, respectivamente, producto de una fermentación homolactica, mostraron valores fisicoquímicamente aceptables para su uso en agricultura.

El Biol además de ser una fuente de nutrientes (N, P, $\mathrm{K}$, Ca, S) también es un fitorregulador de crecimiento porque contiene fitohormonas (Mamani et al., 2012). Bioensayos realizados por Díaz (2017) permitieron confirmar la presencia de sustancias de acción giberélica, auxínica y citocinínica en muestras de bioles de diferentes procedencias. Siendo el bioabono una fuente orgánica de fitoreguladores en pequeñas cantidades es capaz de promover actividades fisiológicas, por ejemplo, induciendo y mejorando la floración y fructificación, activando el vigor y poder germinativo de las semillas. Además, estimula el desarrollo de las plantas favoreciendo el enraizamiento (aumenta $y$ fortalece la base radicular) y ejerce una acción sobre el follaje (amplía la fase foliar), traduciéndose esto en un incremento significativo de la producción de biomasa vegetal (Varnero, 2011; Mamani et al., 2012; Díaz, 2017). Wong y Jimenez (2009), al investigar la aplicación de biofertilizantes líquidos a plantas de algarrobo (Prosopis juliflora) en vivero observaron que el tiempo de permanencia de las plántulas en fase de vivero disminuyó considerablemente de un promedio de 4 meses a 37 días solamente.

A pesar del importante valor de los abonos líquidos en etapas tempranas, debido al bajo contenido de nutrientes de los bioles en los tratamientos $\mathrm{T}_{7}$ (Huallquin-fer) con $0,24 \% \mathrm{~N}, 0,35 \% \mathrm{P}_{2} \mathrm{O}_{5}, 0,19 \%$ $\mathrm{K}_{2} \mathrm{O}$ y $\mathrm{T}_{6}$ (Kapsitron) con $0,27 \% \mathrm{~N}, 0,59 \% \mathrm{P}_{2} \mathrm{O}_{5}$, $0,96 \% \mathrm{~K}_{2} \mathrm{O}$ es que las plantas de café aplicadas con estos productos no alcanzaron su máximo desarrollo. Puede evidenciarse que los contenidos de estos macroelementos fueron los más bajo de todos, sobretodo del Nitrógeno, que es un elemento muy importante en etapa de vivero. Es así que a pesar de los resultados obtenidos con $\mathrm{T}_{7}$ en cuanto a las variables Altura de planta $(16,47 \mathrm{~cm})$ y D Tallo $(2,74 \mathrm{~mm})$, las cuales fueron estadísticamente igual a $\mathrm{T}_{8}$, se pudo evidenciar que las otras variables como $\mathrm{N}^{\circ}$ Hojas $(8,45)$, PF Hojas $(2,09 \mathrm{~g})$, PF Tallo $(0,0043$ g), PF Raíz (0,0060 g) y PF Total $(2,0993$ g) fueron estadísticamente inferiores al $\mathrm{T}_{8} \mathrm{y}$ estadísticamente igual a $\mathrm{T}_{1}$, el testigo sin aplicación de biofertilizantes. Por su parte $\mathrm{T}_{6}$ fue estadísticamente igual a $\mathrm{T}_{8}$ en las variables $\mathrm{N}^{\circ}$ Hojas $(8,65)$, D Tallo $(2,48)$, PF Raíz $(0,0080)$ y estadísticamente inferior a $\mathrm{T}_{8}$ e igual al testigo sin aplicación en las variables PF Hojas (2,11), PF Tallo $(0,0040)$ y PF Total $(2,1230)$.

Finalmente, para todas variables en estudio se evidenció que el Testigo $\left(\mathrm{T}_{1}\right)$, sin aplicación de biofertilizantes sólidos o líquidos, fue estadísticamente inferior en comparación con $\mathrm{T}_{8}$, así como en la mayoría de tratamientos con aplicación de algún biofertilizante, estos resultados concuerdan con Rojas (2017) quien menciona que la aplicación de abonos orgánicos a plantas de café en vivero obtiene una notable influencia en altura de planta y diámetro de tallo sobre testigos sin aplicación, asimismo, Deleon et al. (2015) menciona que entre los macronutrientes requeridos, la baja disponibilidad de nitrógeno es lo más limitante para el desarrollo de las plantas de café; ya que este elemento está asociado con la expansión del área foliar y el crecimiento vegetativo. 
Tabla 2. Efecto de diferentes tipos de biofertilizante en las variables Altura, $\mathrm{N}^{\circ}$ Hojas, Diámetro de Tallo (D Tallo), Peso Fresco de Hojas (PF Hojas), Peso Fresco de Tallo (PF Tallo), Peso Fresco de Raíz (PF Raíz), Peso Fresco Total (PF Total) en plántulas de Café

\begin{tabular}{|c|c|c|c|c|c|c|c|}
\hline Tratamiento & $\begin{array}{c}\text { Altura } \\
(\mathrm{cm})\end{array}$ & $\mathrm{N}^{\circ}$ Hojas & $\begin{array}{c}\text { D Tallo } \\
(\mathrm{mm})\end{array}$ & $\begin{array}{c}\text { PF Hojas } \\
(\mathrm{g})\end{array}$ & $\begin{array}{c}\text { PF Tallo } \\
(\mathrm{g})\end{array}$ & $\begin{array}{c}\text { PF Raíz } \\
(\mathrm{g})\end{array}$ & $\begin{array}{c}\text { PF Total } \\
(\mathrm{g})\end{array}$ \\
\hline $\mathrm{T}_{1}$ & $14,19 \mathrm{e}$ & $7,78 \mathrm{~d}$ & $2,33 \mathrm{~cd}$ & $2,11 \mathrm{bc}$ & $0,0043 \mathrm{~cd}$ & $0,0047 \mathrm{e}$ & $2,0090 \mathrm{bc}$ \\
\hline $\mathrm{T}_{2}$ & $16,61 \mathrm{ab}$ & $8,70 \mathrm{abc}$ & $2,73 \mathrm{abc}$ & $2,47 \mathrm{ab}$ & $0,0047 \mathrm{~cd}$ & $0,0087 \mathrm{abc}$ & $2,4800 \mathrm{~b}$ \\
\hline $\mathrm{T}_{3}$ & 14,84 cde & $8,11 \mathrm{~cd}$ & $2.47 \mathrm{abc}$ & $2,08 \mathrm{bc}$ & $0,0047 \mathrm{~cd}$ & $0,0083 \mathrm{abc}$ & $2,0963 \mathrm{bc}$ \\
\hline $\mathrm{T}_{4}$ & $14,46 \mathrm{de}$ & 8,50 abcd & $2,58 \mathrm{abc}$ & $1,75 \mathrm{c}$ & $0,0047 \mathrm{~cd}$ & $0,0083 \mathrm{abc}$ & $1,9047 \mathrm{c}$ \\
\hline $\mathrm{T}_{5}$ & $17,32 \mathrm{a}$ & $8,9 \mathrm{ab}$ & $2,79 \mathrm{ab}$ & $2,37 \mathrm{~b}$ & $0,0047 \mathrm{~cd}$ & $0,0093 \mathrm{ab}$ & $2,3807 \mathrm{bc}$ \\
\hline $\mathrm{T}_{6}$ & $16,12 \mathrm{~b}$ & $8,65 \mathrm{abc}$ & $2,48 \mathrm{abc}$ & $2,11 \mathrm{bc}$ & $0,0040 \mathrm{~d}$ & 0,0080 abcd & $2,1230 \mathrm{bc}$ \\
\hline $\mathrm{T}_{7}$ & $16,47 \mathrm{ab}$ & $8,45 \mathrm{bcd}$ & $2,74 \mathrm{abc}$ & $2,09 \mathrm{bc}$ & $0,0043 \mathrm{~cd}$ & $0,0060 \mathrm{de}$ & $2,0993 \mathrm{bc}$ \\
\hline $\mathrm{T}_{8}$ & $17,47 \mathrm{a}$ & $9,24 \mathrm{a}$ & $2,89 \mathrm{a}$ & $2,92 \mathrm{a}$ & $0,0073 \mathrm{a}$ & $0,0100 \mathrm{a}$ & $2,9340 \mathrm{a}$ \\
\hline $\mathrm{T}_{9}$ & $16,45 \mathrm{ab}$ & $7,95 \mathrm{~cd}$ & $2,55 \mathrm{abc}$ & $2,26 \mathrm{bc}$ & $0,0043 \mathrm{~cd}$ & $0,0067 \mathrm{~cd}$ & $2,2663 \mathrm{bc}$ \\
\hline $\mathrm{T}_{10}$ & $15,50 \mathrm{bcd}$ & $8,55 \mathrm{abc}$ & $2,61 \mathrm{abc}$ & $2,19 \mathrm{bc}$ & $0,0050 \mathrm{bcd}$ & $0,0077 \mathrm{bcd}$ & $2,2033 \mathrm{bc}$ \\
\hline $\mathrm{T}_{11}$ & $15,73 \mathrm{bc}$ & $8,67 \mathrm{abc}$ & $2.03 \mathrm{~d}$ & $2,28 \mathrm{bc}$ & $0,0057 \mathrm{bc}$ & $0,0083 \mathrm{abc}$ & $2,2917 \mathrm{bc}$ \\
\hline $\mathrm{T}_{12}$ & $14,85 \mathrm{cde}$ & 8,19 bcd & $2,62 \mathrm{abc}$ & $2,32 \mathrm{~b}$ & $0,0043 \mathrm{~cd}$ & $0,0077 \mathrm{bcd}$ & $2,3377 \mathrm{bc}$ \\
\hline $\mathrm{T}_{13}$ & $16,17 \mathrm{~b}$ & $8,66 \mathrm{abc}$ & $2,66 a b c$ & $2,38 \mathrm{~b}$ & $0,0063 a b$ & $0,0070 \mathrm{~cd}$ & $2,3967 \mathrm{bc}$ \\
\hline $\mathrm{T}_{14}$ & $15,55 \mathrm{bcd}$ & 8,17 bcd & $2,48 \mathrm{abc}$ & $2,17 \mathrm{bc}$ & $0,0063 \mathrm{ab}$ & $0,0067 \mathrm{~cd}$ & $2,1790 \mathrm{bc}$ \\
\hline $\mathrm{T}_{15}$ & $15,90 \mathrm{bc}$ & $8,25 \mathrm{bcd}$ & $2,44 \mathrm{bc}$ & $2,25 \mathrm{bc}$ & $0,0040 \mathrm{~d}$ & $0,0083 \mathrm{abc}$ & $2,0957 \mathrm{bc}$ \\
\hline $\mathrm{T}_{16}$ & $15,45 \mathrm{bcd}$ & $8,22 \mathrm{bcd}$ & $2,47 \mathrm{abc}$ & $2,00 \mathrm{bc}$ & $0,0047 \mathrm{~cd}$ & $0,0077 \mathrm{bcd}$ & $2,1787 \mathrm{bc}$ \\
\hline $\mathrm{CV}(\%)$ & 3,90 & 4,60 & 8,57 & 12,50 & 16,67 & 13,57 & 11,43 \\
\hline
\end{tabular}

Letras distintas indican diferencias significativas $(\mathrm{p} \leq 0,05)$, según prueba de Duncan, $\mathrm{T}_{1}=$ Testigo sin biofertilizante, $\mathrm{T}_{2}=$ Churufer (biosol), $\mathrm{T}_{3}=$ Pachacushi (compost), $\mathrm{T}_{4}=$ Guano de Isla, $\mathrm{T}_{5}=$ Churubiol, $\mathrm{T}_{6}=$ Kapsitron (biol), $\mathrm{T}_{7}=$ Huallquin-fer $\quad$ (biol), $\mathrm{T}_{8}=$ Churufer+Churubiol, $\mathrm{T}_{9}=$ Churufer+Kapsitron, $\mathrm{T}_{10}=$ Churufer+Huallquin-fer, $\quad \mathrm{T}_{11}=$ Pachacushi+Churubiol, $\quad \mathrm{T}_{12}=$ Pachacushi $+\quad$ Kapsitron, $\mathrm{T}_{13}=$ Pachacushi+Huallquin-fer, $\mathrm{T}_{14}=$ Guano de Isla+Churubiol, $\mathrm{T}_{15}=$ Guano de Isla+Kapsitron, $\mathrm{T}_{16}=$ Guano de Isla+Huallquin-fer.

\section{CONCLUSIONES}

Los biofertilizantes tienen un efecto positivo en el crecimiento y desarrollo de plantas de café en vivero en comparación con el testigo. El $\mathrm{T}_{8}$ (Churufer+Churubiol) es la combinación de biofertilizantes más efectiva para la producción de plantines de café en vivero. Churufer y Churubiol, obtenidos por medio del proceso de fermentación homoláctica, resultan ser una opción en el manejo de residuos orgánicos generados en fincas cafetaleras lo cual permite darles un valor agregado a los residuos y generar oportunidades económicas y ambientales. Esta innovación generada permitiría que productores cafetaleros orgánicos reduzcan sus costos de producción e incrementen su competitividad en el desarrollo de una agricultura sostenible. Se sugiere determinar la máxima eficiencia de Churufer y Churubiol en el desarrollo vegetativo y productivo de plantas de café en campo definitivo a través del efecto en diferentes concentraciones de los productos.

\section{Conflictos de intereses}

Los autores firmantes del presente trabajo de investigación declaran no tener ningún potencial conflicto de interés personal o económico con otras personas u organizaciones que puedan influir indebidamente con el presente manuscrito.

\section{Contribuciones de los autores}

Preparación y ejecución: JJC, AMV, JJM, EDR, JAG; Desarrollo de la metodología: JJC, AMV, JJM, JJC, AMV, JJM, EDR, JAG; Concepción y diseño: JJC, AMV, JJM, EDR, JAG; Edición del artículo: JJC, AMV, JJM, EDR, JAG; Supervisión del estudio: JJC, AMV, JJM, EDR, JAG. 


\section{LITERATURA CITADA}

- Agro Rural [Programa de Desarrollo Productivo Agrario Rural]. (2018). Plan Anual de Comercialización. 41 pp. Disponible en: https://www.agrorural.gob.pe/wpcontent/uploads/transparencia/documentos/rde/ RDE-124-2018-AG-AGRORURAL-DE.PDF

- Aguirre, E. (2017). Producción de biofertilizante mediante fermentación de la cuyinaza por bacterias del género Lactobacillus aisladas del fermento de la chicha de cebada. Tesis de Ingeniero Ambiental. Universidad Católica Sedes Sapientiae, Lima. Perú. 129 pp.

- Apaza, C. (2018). Inclusión de albúmina de huevo, goma de tara y concentrado funcional de soya en hot dog de llama (Lama glama). Tesis de Magíster Scientiae, Universidad Nacional Agraria La Molina, Lima. Perú. 134 pp.

- Arcáyo, G. (2018). Producción de pellet de lenta liberación a partir de residuos de pulpa de celulosa y poliacrilato de sodio. Tesis de Ingeniero Ambiental, Universidad Nacional Agraria La Molina, Lima. Perú. 98 pp.

- Arizaleta, M., Pire, R., \& Parés, J. (2002). Efecto de la fertilización con N-P-K sobre el contenido foliar y el crecimiento del cafeto (Coffea arabica L.) en la etapa de vivero, en la población de Villanueva, Estado Lara, Venezuela. Rev. Café Cacao, 3(2): 57-61.

- Ascue, J., \& Guerrero, L. (1986). Biogás en el Perú: Situación de la Tecnología, Reciclaje de la Materia Orgánica, Tomo 2, Cajamarca. Perú. 168pp.

- Barrenechea, C. (1986). Café: Problemática y alternativas. Centro Peruano de Estudios Sociales (CEPES), Lima. Perú. 105 pp.

- Belitz, H., \& Grosch, W. (1997). Química de Alimentos. Editorial Acribia S.A., Zaragoza. España. pp. 336-337, 351-355, 884.

- Bonten, L., Zwart, K., Rietra, R., Postma, R., \& de Haas, M. (2014). Bio-slurry as fertilizer; Is bio-slurry from household digester a better fertilizer than manure? A literature review (en línea). Wageningen, Alterra Wageningen UR (University \& Research centre), Alterra report 2519. 46p. Disponible en https://www.researchgate.net/publication/26814 8367_Bioslurry_as_a_fertilizer

- Borjas, R. (2008). Uso de fuentes naturales en la fertilización del café (Coffea arabica) var. Caturra en vivero como base para la producción orgánica en la selva central del Perú. Tesis de Ingeniero Agrónomo, Universidad Nacional Agraria La Molina, Lima. Perú. 82 pp.

- Buchelli, H. (2014). Producción de biofertilizante de bagazo de cebada, excretas de vacuno y suero de quesería mediante fermentación homoláctica. Tesis de Ingeniero Ambiental, Universidad Nacional Agraria La Molina, Lima. Perú. 139 pp.

- $\quad$ Cai, C-T., Cai, Z-Q., Yao, T-Q., \& Qi, X. (2007). Vegetatif growth and photosynthesis in coffee plant under differrent watering and fertilization managements in Yunan, SW China, Photosynthetica, 45(3):455-461.

- Cai, L., Gong, X., Sun, X., Li, S., \& Yu, X. (2018). Comparison of chemical and microbiological changes during the aerobic composting and vermicomposting of green waste. PloS one, 13(11):

- Carhuancho, F. (2012). Aprovechamiento del estiércol de gallina para la elaboración de biol en biodigestores tipo batch como propuesta al manejo de residuo avícola. Tesis Ingeniera Ambiental, Universidad Nacional Agraria La Molina, Lima. Perú. 148 pp.

- Carvajal, J. (1984). Cafeto - Cultivo y Fertilización. Berna, Instituto Internacional de la Potasa. Berna. 254 p.

- Castrillón, J., Morán, T., Prado, E., Zevallos, M. (2017). Planeamiento Estratégico de la Industria Peruana de Café. Tesis de Magister Scientiae. Pontificia Universidad Católica del Perú, Lima. Perú. 190 pp.

- Ceroni, M. (2012). Perú, el país de las oportunidades perdidas en ciencia: el caso de los fertilizantes. Revista de la Sociedad Química del Perú, 78(2): 144-152.

- Cupe, B., \& Juscamaita, J. (2018). Tratamiento de lodos residuales de una industria cervecera a través de fermentación homoláctica para la producción acelerada de abono orgánico. Ecología Aplicada, 17(1): 107-118. 
- Deleon, L., De Souza, L., Tomaz, M., \& Teixeira, J. (2015). The nutritional efficiency of Coffea spp. A review. Academic Journals, 14(9): 728-734.

- Díaz, A. $\left(2017^{a}\right)$. Características físicoquímicas y microbiológicas del proceso de elaboración de biol y su efecto en germinación de semillas. Tesis de Magister Scientiae. Universidad Nacional Agraria La Molina, Lima. Perú. 129 pp.

- Evangelista, A., Júnior, J., \& Melo, P. (2013). Resposta do cafeeiro à aplicação de níveis de irrigação e adubação com Alfertil. Revista Brasileira de Engenharia Agrícola e Ambiental, 17(4): 392-396.

- Herrera, R. (2017). Elaboración de un abono líquido a partir de residuos de fresa (Fragaria $\mathrm{X}$ Ananassa) por fermentación láctica. Tesis Ingeniera Ambiental, Universidad Nacional Agraria La Molina, Lima. Perú. 104 pp.

- Koehbach, J., \& Gruber, C. (2015). Chapter Three - Cyclotides in the Rubiaceae. Advances in Botanical Research, 76: 51-78.

- Malavolta, E. (2000). História Do Café No Brasil. Editora Agronomica Ceres. Sao Paulo. $456 \mathrm{p}$.

- Mamani, P., Chavez, E., \& Ortuño, N. (2012). El biol - Biofertilizante casero para la producción ecológica de cultivos. Fundación PROINPA. Disponible en: https://www.proinpa.org/tic/pdf/Bioinsumos/Bi ol/pdf59.pdf

- Mancilla, J. (2012). Factibilidad técnicaeconómica de una planta de carragenina kappa I. Tesis de Ingeniero Civil Químico. Pontificia Universidad Católica de Valparaíso. Chile. 216 pp.

- Maradiaga, W., Pego, A., Alves J., \& Vinicius, M. (2017). Producción de plántulas café cultivado en diferentes tipos de substratos y fertilizado con lithothamnium. Rev.Fac.Nac.Agron. 70(2): 8177-8182.

- Marcuzzo, K., Melo, B., Carvalho, H., Teodoro, E., \& Alvarenga, B. (2005). Desenvolvimento de mudas de cafeeiro em diferentes substratos e doses de fertilizante de liberaçao gradual. Bioscience Jornal, 21(57):57-63.
- $\quad$ Medeiros, B.; Lopes, S. 2006. Biofertilizantes líquidos e sustentabilidade agrícola. Bahia Agricola, 7(3): 24-32.

- Medeiros, C., Strassburger, A., \& Antunes, L. (2008). Avaliação de substratos constituídos de cascas de arroz no cultivo sem solo de morangueiro. Características químicas de solos sob diferentes usos na Bacia do Rio Piranhas. Horticultura Brasileira ,26(2): 827-831.

- Medina, A., Quipuzco, L., \& Juscamaita, J. (2015). Evaluación de la calidad de biol de segunda generación de estiércol de ovino producido a través de biodigestores. Anales Científicos, 76 (1): 116-124.

- Meza, L. (2014). Elaboración de abono líquido mediante fermentación homoláctica de papas de descarte utilizando el consorcio microbiano ácido láctico B-lac. Tesis de Biólogo, Universidad Nacional Agraria La Molina, Lima. Perú. 134 pp.

- $\quad$ MINAGRI [Ministerio de Agricultura y Riego]. (2018). Plan Nacional de Acción del Café Peruano, 2018-2030, Lima. Perú. 54 pp.

- MINAGRI [Ministerio de Agricultura y Riego]. (2019). Boletin Producción y Comercio de la TARA en el Perú. Disponible en: http://agroaldia.minagri.gob.pe/biblioteca/down load/pdf/tematicas/f-taxonomia_plantas/f01cultivo/2019/produccion_comercio_de_tara.pdf

- MINCETUR [Ministerio de Comercio Exterior y Turismoo3. (2018). Expo Café Perú 2018 reunirá participantes de 15 países. Disponible en: https://www.gob.pe/institucion/mincetur/noticia s/17958-expo-cafe-peru-2018-reuniraparticipantes-de-15-paises

- Montoya, S. (2007). Industrialización de la yuca. Obtención de almidón nativo y sus aplicaciones. Universidad del Valle, Cali. Colombia. 40 pp.

- Muliasari, A., Wachjar, A., \& Supijatno. (2015). The Growth of Arabica Coffee (Coffea arabica L.) Seedling on Combination of Inorganicorganic Fertilizers and Shading Level. Asian Journal of Applied Sciences, 3: 739-746.

- Peralta, L., Juscamaita, J., \& Meza, V. (2016). Obtención y caracterización de abono orgánico líquido a través del tratamiento de excretas del ganado vacuno de un establo lechero usando un 
consorcio microbiano ácido láctico. Ecología Aplicada, 15(1): 1-10.

- $\quad$ Pindi, P. (2012). Liquid Microbial Consortium. A Potential Tool for Sustainable Soil Health. Journal of Fertilizers \& Biopesticides, 3 (4): 1-9.

- Pinedo, M., Abanto-Rodríguez, C., Oroche, D., Paredes, E., Bardales-Lozano, R., Alves, E., Lopes, J., \& Vargas, J. (2018). Mejoramiento de las características agronómicas y rendimiento de fruto de camu-camu con el uso de biofertilizantes en Loreto, Perú. Scientia Agropecuaria, 9(4): $527-533$.

- Quiñones, H., Trejo, W., \& Juscamaita, J. (2016). Evaluación de la calidad de un abono líquido producido vía fermentación homoláctica de heces de alpaca. Ecología Aplicada, 15 (2): 133-142.

- $\quad$ Rezende, R., Junior, C., Souza, R., Antunes, F., \& Frizzone, J. (2010). Crescimento inicial de duas cultivares de cafeeiro em diferentes regimenes hídricos e dosagens de fertirrigaçao. Revista Engenharia Agrícola, 30(3): 447-458.

- Rojas, K. (2017). Enmiendas orgánicas (guano de isla, humus y compost) en Coffea Arabica L., variedad costa rica 95 en condiciones de vivero - en el fundo Be Hurt Satipo- Perú. Tesis de Ingeniero Agrónomo, Universidad Nacional del Centro del Perú, Satipo. Perú. 49 pp.

- Román, C. (2012). Tratamiento biológico de la cuyinaza a través de un proceso de fermentación homoláctica. Tesis de Ingeniero Ambiental, Universidad Nacional Agraria La Molina, Lima. Perú. 207 pp.

- SENASA [Servicio Nacional de Sanidad Agraria]. (2006). Decreto Supremo N044-2006AG. Reglamento Técnico para los Productos Orgánicos. Accedido en https://www.senasa.gob.pe/senasa/normassobre-produccion-organica/

- Shah Jahan Leghari, Niaz Ahmed Wahocho, Ghulam Mustafa Laghari, Abdul HafeezLaghari, Ghulam MustafaBhabhan, Khalid HussainTalpur, Tofique Ahmed Bhutto, Safdar Ali Wahocho, \& Ayaz Ahmed Lashari. (2016). Role of Nitrogen for Plant Growth and Development: A review. Advances Environmental Biology, 10(9): 209-218.
- USDA [United States Deparment of Agriculture]. (2013). AMS National Organic Program. Reglamentos Orgánicos Estadounidenses. Disponible en: https://www.ams.usda.gov/sites/default/files/me dia/NOP-

ReglamentosOrganicosEstadounidenses.pdf

- USDA [United States Deparment of Agriculture]. (2018). Foreign Agricultural Service. Coffee Anual. Gain Report. Global Agricultural Information. Disponible en: https://gain.fas.usda.gov/Recent\%20GAIN\%20 Publications/Coffee\%20Annual_Lima_Peru_59-2018.pdf

- Varnero, M. (2011). Manual de biogás. Organización de las Naciones Unidas para la Alimentación y la Agricultura (FAO), Ministerio de Energía (MINENERGIA), Programa de las Naciones Unidas para el Desarrollo (PNUD), Global Enviroment Facility (GEF). Santiago de Chile. 120 pp. Disponible en: www.fao.org/docrep/019/as400s/as400s.pdf

- Wong, M., \& Jimenez, E. (2009). Comparación del efecto de 2 biofertilizantes líquidos a base de estiércol caprino y vacuno sobre parámetros de crecimiento de algarrobo (Prosopis juliflora (Sw.) DC) en fase de vivero. Escuela Superior Politécnica del Litoral, Guayaquil. Ecuador. 